\title{
Glucose tolerance and other determinants of cardiovascular autonomic function: the Hoorn Study
}

\author{
J. Gerritsen ${ }^{1}$, J.M.Dekker ${ }^{2}$, B.J. TenVoorde ${ }^{1}$, F.W.Bertelsmann ${ }^{3}$, P.J.Kostense ${ }^{4}$, C.D.A.Stehouwer ${ }^{2}$, R.J.Heine ${ }^{2}$, \\ G. Nijpels ${ }^{2}$, R.M.Heethaar ${ }^{1}$, L.M. Bouter ${ }^{2}$
}

${ }^{1}$ Department of Clinical Physics and Informatics, Vrije Universiteit, Amsterdam, The Netherlands

${ }^{2}$ Institute for Research in Extramural Medicine, Vrije Universiteit, Amsterdam, The Netherlands

${ }^{3}$ Department of Neurology, Hospital Amstelveen, Amstelveen, The Netherlands

${ }^{4}$ Department of Clinical Epidemiology and Biostatistics, Vrije Universiteit, Amsterdam, The Netherlands

\section{Abstract}

Aims/hypothesis. Currently, three categories of measures are used to assess cardiovascular autonomic dysfunction: measures of the Ewing-test, measures of heart-rate variability, and measures of baroreflex sensitivity. We studied the determinants of these measures obtained from cardiovascular autonomic function tests in the Hoorn Study.

Methods. The study group $(n=631)$ consisted of a glucose-tolerance-stratified sample from a 50- to 75year-old group of people. Cardiac cycle duration ( RR interval) and continuous finger arterial pressure were measured under three conditions: during (a) spontaneous breathing, (b) six deep breaths over one minute, and (c) an active change in position from lying to standing. From these readings, ten measures of autonomic function were assessed (three Ewing, six heart-rate variability and one baroreflex sensitivity). As possible determinants we considered age, sex, glucose tolerance, cardiovascular disease, use of anti-hypertensive drugs, anthropometric factors, metabolic factors and lifestyle factors.
Results. Multivariate analysis showed that eight of ten cardiovascular autonomic function measures were most strongly associated with glucose tolerance. Furthermore, measures were moderately associated with age, sex, waist-to-hip ratio, use of anti-hypertensive drugs, and insulin. The measures were weakly associated with coronary artery disease but not with lipids. The strongest determinants seemed to differ between subjects with and without diabetes: in the non-diabetic subjects the most strongly associated were age and use of anti-hypertensive drugs and in subjects with diabetes, insulin. No consistent differences in association between the three categories of measures were observed.

Conclusion/interpretation. The strongest determinants of autonomic function were age, presence of diabetes and use of anti-hypertensive drugs. [Diabetologia (2000) 43: 561-570]

Keywords Aging, baroreflex, Type II diabetes mellitus, cardiovascular disease, glucose intolerance, heart-rate variability, hypertension, lifestyle, autonomic nervous system, obesity.
Received: 25 October 1999 and in revised form: 3 January 2000

Corresponding author: J. Gerritsen, Department of Clinical Physics and Informatics, Faculty of Medicine, Vrije Universiteit, P. O. Box 7057, 1007 MB Amsterdam, The Netherlands Abbreviations: BRS, Baroreflex sensitivity (ms/mmHg); DM, diabetes mellitus; EI difference, expiration-inspiration difference in RR intervals during breathing at 6/min (ms); HRV, heart-rate variability; HF power, high frequency power in the RR-interval spectrum between $0.12-0.40 \mathrm{~Hz}\left(\mathrm{~ms}^{2}\right)$; KDM, known diabetes mellitus; LF power, low frequency power in the RR-interval spectrum between $0.04-0.12 \mathrm{~Hz}\left(\mathrm{~ms}^{2}\right)$; LF/
$(\mathrm{LF}+\mathrm{HF})$, ratio of low frequency power to the sum of low and high frequency power in the RR-interval spectrum; Mean $\mathrm{NN}$, mean of all sinus rhythm (normal-to-normal) RR intervals (ms); NDM, newly-diagnosed diabetes mellitus; RRmax, maximal change in RR interval after standing up (ms); $\mathrm{RRmax} / \mathrm{min}$, maximal RR interval between $15 \mathrm{~s}$ and $30 \mathrm{~s}$ after standing up divided by the minimal RR interval within $15 \mathrm{~s}$ after standing up; SBP difference, systolic blood pressure 1.5-2 min after standing up minus systolic blood pressure in supine position $(\mathrm{mmHg})$; SDNN, standard deviation of all sinus rhythm (normal-to-normal) RR intervals (ms). 
Standard cardiovascular autonomic function tests, such as the deep breathing test, the lying-to-standing test, the Valsalva manoeuvre and the sustained handgrip test, also known as the 'Ewing battery', still are the corner-stones for the diagnosis of diabetic cardiovascular autonomic neuropathy [1-3]. During the last decade, other measures have come into use, notably heart-rate variability (HRV) and baroreflex sensitivity (BRS) $[4,5]$. Besides their application in the assessment of cardiovascular autonomic function in diabetic patients, these new measures have been introduced in cardiology for risk stratification after myocardial infarction. Low HRV is associated with mortality risk in post-myocardial infarction patients $[6$, 7] and also in the general population $[8,9]$.

Diabetic patients have lower values of the Ewing battery [1], of the spectral analysis of HRV [10, 11], and have a low BRS $[5,12]$ in comparison with normal glucose tolerant control subjects. Besides diabetes, many other factors possibly influence the outcome of cardiovascular autonomic function tests, including age [13-15], sex [16], anthropometric factors [17], cardiovascular disease [18, 19], use of anti-hypertensive drugs [20, 21], metabolic factors [22-24], and lifestyle $[25,26]$. Since most of these associations have been described in persons without diabetes, it is not known to what extent such factors contribute to the severity of autonomic dysfunction in diabetic subjects. Furthermore, the strength of the relation between several risk factors (serum cholesterol, systolic blood pressure and cigarette smoking) and death from cardiovascular origin has been reported to be significantly modified (i.e. stronger) by diabetes in the Multiple Risk Factor Intervention Trial (MRFIT) [27]. Therefore, it is of interest to study the possibility of effect-modification by glucose tolerance on the associations of cardiovascular autonomic function with the determinants identified in the non-diabetic domain.

Qualitative and quantitative knowledge about the association between test measures and the putative determinants is useful for pathological conceptualisation and possibly also for clinical practice. Although various determinants have been studied separately, the effect of these factors together, and on a variety of autonomic function measures, to our knowledge has not been established in relation to glucose intolerance. We studied this in the Hoorn Study in which we investigated 50- to 75-year-old Caucasian subjects.

\section{Subjects and methods}

Design and study group. This study is part of the Hoorn Study, a prospective study on glucose tolerance and other cardiovascular risk factors in a 50- to 75-year-old cohort [28]. The baseline examination was carried out from October 1989 until February 1992, as previously described in detail [29]. Briefly, a random sample of all men and women born between 1914 and
1940 was drawn from the municipal population of the town of Hoorn, the Netherlands; 2484 persons participated (71\%). All subjects had a 75-g oral glucose tolerance test (OGTT), except those diagnosed previously with diabetes, as indicated by treatment with oral glucose-lowering drugs or insulin. Stratified by the 2-h glucose concentrations of the first test, age and sex, 708 subjects were invited for extensive examinations of diabetes-related complications. Of this sample 631 (89\%) participated and thus became the study group. The study protocol was approved by the ethics committee of the University Hospital of the Vrije Universiteit. All study participants gave their informed consent.

Putative determinants. Fasting and 2-h post-load venous plasma glucose concentrations were determined with a glucose dehydrogenase method (Merck, Darmstadt, Germany). Subjects were classified into glucose tolerance groups according to the criteria of the World Health Organization (WHO), based on the mean values of two OGTTs, 3 to 5 weeks apart from each other [30]. We established that 288 subjects had normal glucose tolerance (NGT), 169 had impaired glucose tolerance (IGT), 95 had newly diagnosed diabetes (NDM), and 79 subjects were known Type II (non-insulin-dependent) diabetic patients (KDM) as defined by the use of a diet, oral glucose-lowering drugs or insulin.

Glycated haemoglobin $\left(\mathrm{HbA}_{1 \mathrm{c}}\right)$ was determined by ion-exchange high-performance liquid chromatography, using a Modular Diabetes Monitoring System (Bio-Rad, Veenendaal, The Netherlands).

Height, weight and body circumferences were measured on all subjects barefoot and wearing only light clothing. Body mass index (BMI, $\mathrm{kg} / \mathrm{m}^{2}$ ) was calculated as weight divided by height squared. Waist-to-hip ratio (WHR) was defined as waist circumference divided by hip circumference.

Cardiovascular disease was defined as coronary artery, cerebrovascular or peripheral arterial disease. The self-reported cardiovascular disease history was obtained by means of a questionnaire. Subjects were asked whether they had received any medical care during the past 10 years and, if so, for what reason and from which specialty. The history of macrovascular disease (myocardial infarction, angina pectoris, intermittent claudication, transient ischemic attack, or stroke) was assessed by means of a Dutch translation of the Rose questionnaire [31]. Furthermore, the names of the drugs and dosages prescribed were recorded. Coronary artery disease was defined as a self-reported history of myocardial infarction, self-reported angina pectoris, use of nitrates, coronary artery bypass grafting or Minnesota codes 1-1 or 1-2 on the ECG. Peripheral arterial disease was defined as a self-reported peripheral arterial reconstruction, self-reported intermittent claudication, non-traumatic limb amputation, an ankle brachial pressure index (ABPI) less than 0.90 or a monophasic/absent flow curve in the left or right leg arteries as assessed by Doppler flow velocity measurements [32]. The ABPI was obtained by means of Doppler-assisted systolic blood pressure measurements taken from the brachial and the three crural arteries on both sides as described previously in more detail [32]. The lowest ABPI of either limb was used for statistical analysis. Cerebrovascular disease was defined as a self-reported history of transient ischaemic attack, stroke or a carotid stenosis greater than $80 \%$ as assessed by ultrasonographic examination of both common, internal and external carotid arteries, done by means of a colour-coded Duplex-Doppler scanner (Acuson 128, Mountain View, Calif., USA). We classified subjects into two categories on the basis of the maximum percentage of stenosis of the more diseased of the two carotid arteries: 0 to $80 \%$ or 81 to $100 \%$. 
Table 1. Overview of the ten cardiovascular autonomic functions measures classified into three categories according to involvement of relatively more cardiac vagal, more cardiac sympathetic or more peripheral vasomotor sympathetic functioning

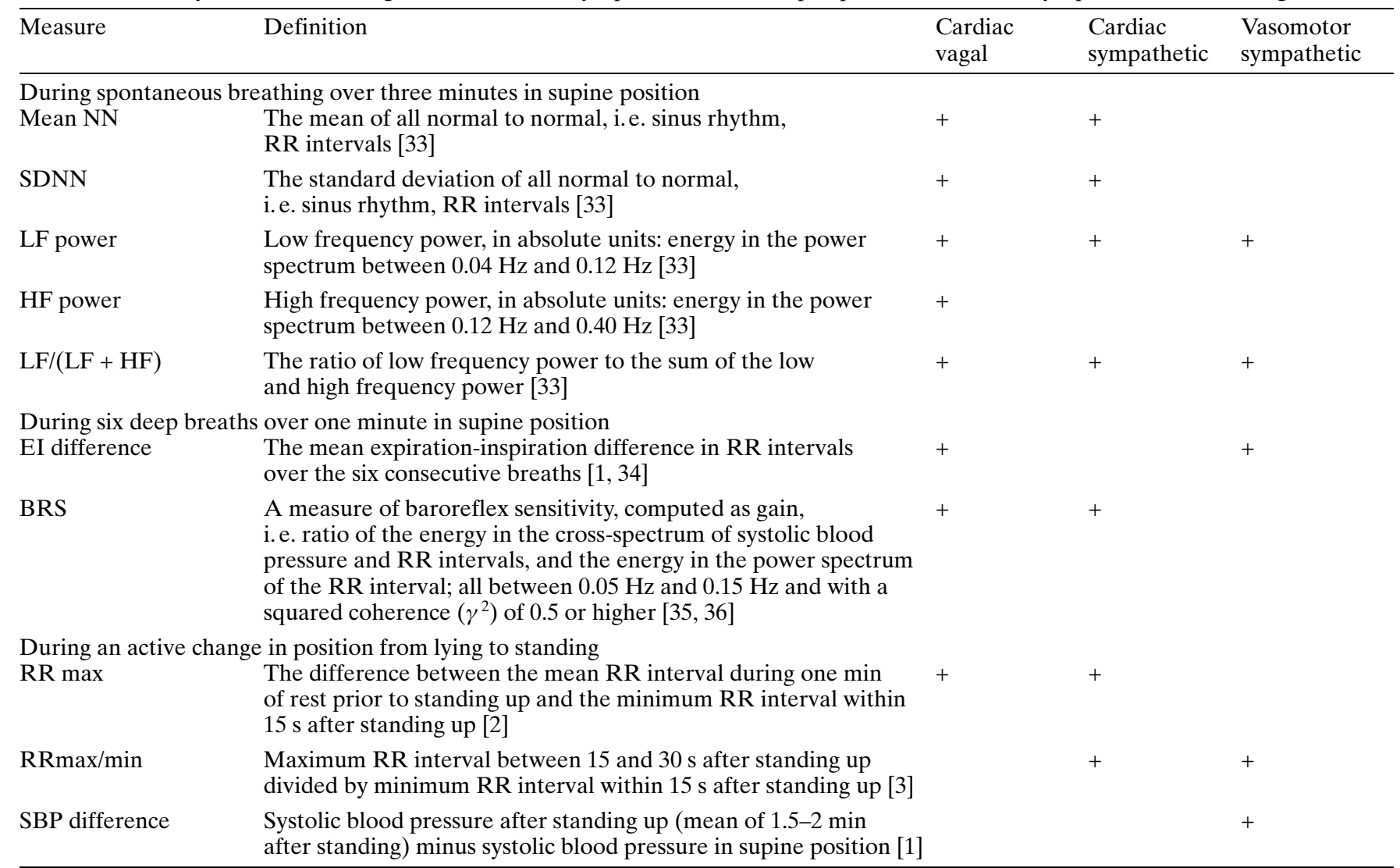

Use of anti-hypertensive drugs was defined as current treatment with alpha blockers, beta blockers, calcium antagonists, angiotensin converting enzyme inhibitors, diuretics, and/or centrally acting anti-hypertensive drugs.

Blood pressure was measured twice on two occasions on the right arm of seated subjects after they had rested for at least $5 \mathrm{~min}$, by means of a random zero mercury sphygmomanometre (Hawksley-Gelman, Lancing, Sussex, UK). The average of the four readings of systolic (SBP) and diastolic blood pressure (DBP) (Korotkov V) was calculated. Hypertension on the basis of blood pressure was thus defined as a SBP of $160 \mathrm{mmHg}$ or higher and/or a DBP of $95 \mathrm{mmHg}$ or higher. Furthermore, hypertension according to the World Health Organization was defined as use of anti-hypertensive drugs or hypertension on the basis of blood pressure or both.

Fasting specific serum insulin concentrations were quantified with an insulin specific double antibody radioimmunoassay (antibody: Linco SP21, St. Louis, Mo., USA), without cross-reactivity with proinsulin or split proinsulin.

Concentrations of serum lipids were determined in the fasting blood sample of all subjects. Total cholesterol, HDL cholesterol and triglycerides were measured by an enzymatic procedure (Boehringer-Mannheim, Mannheim, Germany).

The information on lifestyle variables was obtained by means of questionnaires. Physical activity was calculated as the number of positive answers to nine questions concerning activity (e.g. cycling, walking staircases). Cigarette smoking and alcohol consumption were expressed on a dichotomized scale: current smokers, current alcohol consumers vs non-users.
Cardiovascular autonomic function tests. For the assessment of cardiovascular autonomic function, participants were asked to refrain from smoking and drinking coffee for $2 \mathrm{~h}$ prior to the visit to the research centre. Tests were done between 0830 and 1600 hours at least $1 \mathrm{~h}$ after a light meal. A quiet setting was ensured, with a room temperature between 19 and $22^{\circ} \mathrm{C}$. All tests on all subjects were carried out by a single investigator who was blinded to the glucose tolerance status. Cardiac cycle duration ( $\mathrm{RR}$ interval) and continuous finger arterial pressure were measured under three conditions: (a) during spontaneous breathing over $3 \mathrm{~min}$ in the supine position, (b) during six deep breaths over $1 \mathrm{~min}$ in the supine position, and (c) during an active change in position from lying to standing. The frequency of breathing at six breaths per min was dictated by the investigator. When off-line spectral analysis showed that breathing was not done at the appropriate frequency, the record was discarded. The test session started with a resting period of at least $10 \mathrm{~min}$, and each separate test started with a resting period of at least $1 \mathrm{~min}$. During the tests, RR intervals and blood pressure were continuously recorded on a PC-based data-acquisition system. We obtained RR intervals from an electrocardiogram by a hardware QRS detector with an accuracy of one millisecond. Blood pressure was recorded using the Finapres method (Finger Arterial Blood Pressure, Ohmeda BP2000, Englewood, Colo., USA). We obtained SBP values from the $200 \mathrm{~Hz}$ sampled continuous blood pressure signal by means of an automatic procedure, which was verified by visual inspection.

Individual data were missing for the following reasons: the test schedule was not completed, the quality of the data was in- 
Table 2. Characteristics of the study group stratified for glucose tolerance

\begin{tabular}{|c|c|c|c|c|c|}
\hline & & \multicolumn{4}{|c|}{ Glucose tolerance } \\
\hline & & $\begin{array}{l}\text { NGT } \\
n=288\end{array}$ & $\begin{array}{l}\text { IGT } \\
n=169\end{array}$ & $\begin{array}{l}\text { NDM } \\
n=95\end{array}$ & $\begin{array}{l}\text { KDM } \\
n=79\end{array}$ \\
\hline $\begin{array}{l}\text { ANTHROPOMETRIC FACTORS } \\
\text { BMI }\left(\mathrm{kg} / \mathrm{m}^{2}\right) \\
\text { WHR }\end{array}$ & & $\begin{array}{l}26.0 \pm 3.3 \\
0.90 \pm 0.08\end{array}$ & $\begin{array}{l}27.8 \pm 3.7^{\mathrm{b}} \\
0.93 \pm 0.08^{\mathrm{b}}\end{array}$ & $\begin{array}{l}28.8 \pm 4.2^{\mathrm{b}} \\
0.96 \pm 0.09^{\mathrm{b}}\end{array}$ & $\begin{array}{l}28.8 \pm 5.0^{\mathrm{b}} \\
0.94 \pm 0.08^{\mathrm{b}}\end{array}$ \\
\hline $\begin{array}{l}\text { LIFESTYLE FACTORS } \\
\text { Physical Activity Score (0-9) } \\
\text { Smoking } \\
\text { Alcohol consumption }\end{array}$ & $\begin{array}{l}\text { yes }(\%) \\
\text { yes }(\%)\end{array}$ & $\begin{array}{l}5.50 \pm 1.49 \\
86(30) \\
207(72)\end{array}$ & $\begin{array}{c}5.14 \pm 1.66^{\mathrm{c}} \\
40(24) \\
97(57)^{\mathrm{c}}\end{array}$ & $\begin{array}{c}5.07 \pm 1.76^{\mathrm{c}} \\
19(20) \\
59(62)\end{array}$ & $\begin{array}{c}4.85 \pm 1.73^{\mathrm{c}} \\
20(25) \\
36(46)^{\mathrm{b}}\end{array}$ \\
\hline
\end{tabular}

Values are means \pm SD or number of subjects and percentage between brackets. ${ }^{\mathrm{a}}$ no testing (stratified), ${ }^{\mathrm{b}} p<0.001$ compared to the NGT group, ${ }^{\mathrm{c}} p<0.05$ compared to the NGT group

sufficient for processing (a poor blood pressure signal or arrhythmias) or there were more than $10 \%$ non-sinus beats in the total number of recorded beats.

Test parameters. We computed ten measures of cardiovascular autonomic function from the RR interval and the SBP recordings under the three respective conditions. Table 1 gives the definition and the computational information of the ten measures [1-3, 33-36].

Statistical analysis. All analyses were done with SPSS 7.5 for Windows 95. Differences between the IGT vs NGT, NDM vs NGT and KDM vs NGT of the continuous variables were assessed by analysis of variance, and the analogous differences in proportions were tested with a Chi-squared test. Additionally, for the measures of autonomic function, the variation due to regression was tested (F-test) to assess whether a trend with glucose tolerance was present. Because of the skewed distributions, SDNN, LF power, HF power, EI difference, BRS, $\mathrm{RRmax}$, and RRmax/min values were logarithmically transformed. Geometric means were computed by back transformation of the means of logarithmically transformed values.

To estimate the effects of all putative determinants on the test measures, multiple linear and logistic regression analysis were done. For the linear regression analysis, logarithmic transformation of SDNN, LF power, HF power, EI difference, BRS, RRmax, and RRmax/min were necessary to obtain normally distributed residuals. For logistic regression the lowest 25 th percentile values of the autonomic function measures in the NGT group were taken as cut-offs. In this case the odds ra- tio resulting from the logistic analyses can be interpreted as a relative risk. A relative risk of 1 indicates that there is no association between the determinants and the occurrence of autonomic dysfunction, a relative risk greater than 1 indicates a risk factor, and a relative risk less than 1 (and $\geq 0$ ) indicates a protective factor. Because the study group was stratified by age, sex and glucose tolerance, these three variables were entered into the regression model (the 'basic' model), even when they did not significantly contribute after other variables were entered. Subsequently, each putative determinant was entered separately into the 'basic' model. Then four models were constructed: the 'cardiovascular', the 'metabolic', the 'life-style' model, and the 'full' model including all determinants. As putative determinants we considered for the 'cardiovascular' model: coronary artery disease (yes/no), cerebrovascular disease (yes/no), peripheral arterial disease (yes/no), hypertension on the basis of blood pressure (yes/no), and use of antihypertensive drugs (yes/no). For the 'metabolic' model: $\mathrm{BMI}$, WHR, fasting glucose, $\mathrm{HbA}_{1 \mathrm{c}}$, fasting insulin, total cholesterol, HDL cholesterol, and triglycerides (all continuous). For the 'life-style' model: physical activity (score 0-9), smoking (yes/no), and use of alcohol (yes/no). The analyses were done for the total group and also separately for each glucose tolerance group to explore whether there were differences in the putative determinants according to glucose tolerance. The influence of glucose tolerance on the observed associations, i. e. effect modification by glucose tolerance, was further tested by adding product terms to the regression model. 
Table 3. (Geometric) means and 95-per cent confidence intervals for ten measures of cardiovascular autonomic function stratified for glucose tolerance

\begin{tabular}{|c|c|c|c|c|c|}
\hline Measure & $\begin{array}{l}\text { NGT } \\
n=288\end{array}$ & $\begin{array}{l}\text { IGT } \\
n=169\end{array}$ & $\begin{array}{l}\text { NDM } \\
n=95\end{array}$ & $\begin{array}{l}\text { KDM } \\
n=79\end{array}$ & Test for trend \\
\hline Mean NN (ms) & $\begin{array}{l}970 \\
{[952 \text { to } 988]}\end{array}$ & $\begin{array}{l}945 \\
{[921 \text { to } 969]}\end{array}$ & $\begin{array}{l}901^{\mathrm{b}} \\
{[866 \text { to } 936]}\end{array}$ & $\begin{array}{l}885^{\mathrm{b}} \\
{[855 \text { to } 916]}\end{array}$ & $p<0.01$ \\
\hline LF power ${ }^{\mathrm{a}}\left(\mathrm{ms}^{2}\right)$ & $\begin{array}{l}251 \\
{[220 \text { to } 286]}\end{array}$ & $\begin{array}{l}224 \\
{[190 \text { to } 264]}\end{array}$ & $\begin{array}{l}163^{\mathrm{b}} \\
{[123 \text { to } 215]}\end{array}$ & $\begin{array}{l}148^{\mathrm{b}} \\
{[109 \text { to } 202]}\end{array}$ & $p<0.01$ \\
\hline $\mathrm{LF} /(\mathrm{LF}+\mathrm{HF})$ & $\begin{array}{l}0.55 \\
{[0.52 \text { to } 0.57]}\end{array}$ & $\begin{array}{l}0.56 \\
{[0.42 \text { to } 0.59]}\end{array}$ & $\begin{array}{l}0.54 \\
{[0.49 \text { to } 0.58]}\end{array}$ & $\begin{array}{l}0.56 \\
{[0.52 \text { to } 0.61]}\end{array}$ & $p>0.05$ \\
\hline EI difference ${ }^{\mathrm{a}}(\mathrm{ms})$ & $\begin{array}{l}165 \\
{[154 \text { to } 177]}\end{array}$ & $\begin{array}{l}148 \\
{[135 \text { to } 163]}\end{array}$ & $\begin{array}{l}127^{\mathrm{b}} \\
{[110 \text { to } 146]}\end{array}$ & $\begin{array}{l}121^{\mathrm{b}} \\
{[105 \text { to } 140]}\end{array}$ & $p<0.01$ \\
\hline $\mathrm{BRS}^{\mathrm{a}}(\mathrm{ms} / \mathrm{mm} \mathrm{Hg})$ & $\begin{array}{l}8.0 \\
{[7.5 \text { to } 8.6]}\end{array}$ & $\begin{array}{l}7.2 \\
{[6.5 \text { to } 8.0]}\end{array}$ & $\begin{array}{l}6.1^{\mathrm{b}} \\
{[5.3 \text { to } 7.0]}\end{array}$ & $\begin{array}{l}6.3^{\mathrm{b}} \\
{[5.4 \text { to } 7.5]}\end{array}$ & $p<0.01$ \\
\hline $\mathrm{RRmax} / \min ^{\mathrm{a}}$ & $\begin{array}{l}1.26 \\
{[1.24 \text { to } 1.28]}\end{array}$ & $\begin{array}{l}1.24 \\
{[1.21 \text { to } 1.26]}\end{array}$ & $\begin{array}{l}1.19^{\mathrm{b}} \\
{[1.16 \text { to } 1.22]}\end{array}$ & $\begin{array}{l}1.19^{\mathrm{b}} \\
{[1.16 \text { to } 1.22]}\end{array}$ & $p<0.01$ \\
\hline SBP difference $(\mathrm{mm} \mathrm{Hg})$ & {$[-4.5$} & $\begin{array}{l}-6.6 \\
{[-8.8 \text { to }-4.4]}\end{array}$ & {$[-5.5$} & $\begin{array}{l}-10.1^{\mathrm{b}} \\
{[-13.8 \text { to }-6.3]}\end{array}$ & $p<0.05$ \\
\hline
\end{tabular}

${ }^{\mathrm{a}}$ Geometric means are given; ${ }^{\mathrm{b}} p<0.05$ compared to the NGT group, adjusted for age and sex

\section{Results}

Table 2 shows the characteristics of the study group in four categories of glucose tolerance. On average, persons with IGT and diabetes were older, more often had a history of cardiovascular disease, and had an adverse cardiovascular risk profile.

Table 3 shows the (geometric) means of the ten autonomic function measures for each glucose tolerance group. After adjustment for age and sex, diabetic patients still had statistically significant lower values for all studied measures of autonomic function, except LF/(LF + HF) (Table 3). Persons with IGT had significantly lower values for SDNN compared with the NGT persons; all other measures of autonomic function were also lower in the IGT group, but this was not statistically significant. Also, after adjustment for age and sex, a trend was observed in the relation with glucose tolerance, except for $\mathrm{LF} /$ $(\mathrm{LF}+\mathrm{HF})$ (Table 3, test for trend).

Basic regression model including each putative determinant separately (the complete group). For the complete group (NGT, IGT, NDM and KDM together) age was negatively associated with most measures of autonomic function, except for the Mean NN, LF/ $(\mathrm{LF}+\mathrm{HF})$, and SBP difference (Table 4). Sex also appeared to be a determinant of cardiovascular autonomic function: $\mathrm{LF}$ power, $\mathrm{LF} /(\mathrm{LF}+\mathrm{HF}), \mathrm{RRmax}$, and SBP difference were lower in women. Furthermore, adjusted for age, sex, and glucose tolerance, a consistent association - for five of the ten measures of autonomic function - was found for the use of anti-hypertensive drugs. Additional analyses showed that the observed associations were mainly attributable to the use of beta-blockers and diuretics. Less consistent associations - one to three of the ten measures of autonomic function - were found for coronary artery disease, cerebrovascular disease, hypertension on the basis of blood pressure, BMI, WHR, and fasting insulin.

Basic, cardiovascular, metabolic and life-style regression model (for each glucose tolerance group). Both linear and logistic regression within the four glucose tolerance groups showed that the observed associations in the total group were dependent on glucose tolerance, i. e. effect-modification: the observed associations in the total group were sometimes completely attributable to an association in only one of the four glucose tolerance groups. Furthermore, these findings were confirmed as being statistically significant by adding the appropriate product terms in the regression models (for the complete group). There was a strong association of SDNN, LF power, HF power, EI difference, BRS and RRmax with age in the NGT and IGT group, whereas for the NDM and KDM the association with age was rather weak and statistically non-significant (Fig. 1). The product terms for SDNN, LF power, HF power and EI difference with age were statistically significant $(p<0.10)$. Only for RRmax/min was the association with age 


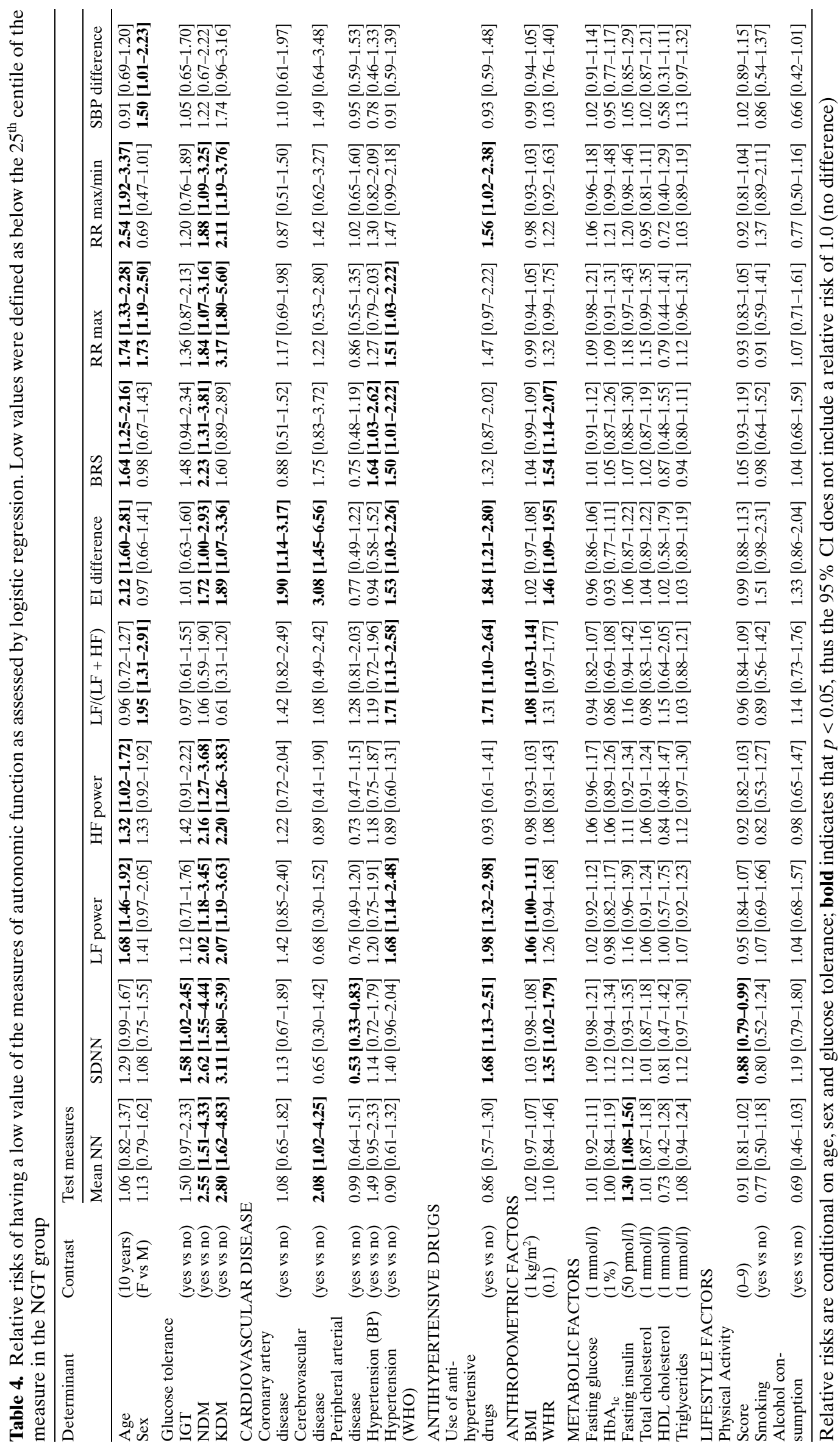




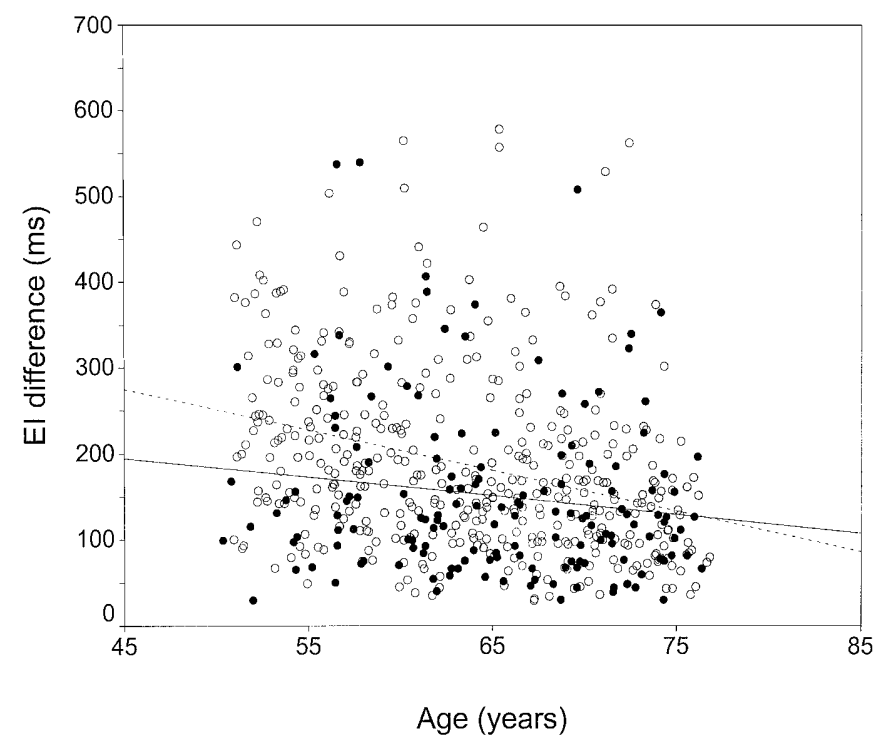

Fig. 1. Scatterplot of EI difference and age, stratified for glucose tolerance. $\bigcirc$, dotted line, non-diabetic subjects (normal and impaired glucose tolerant); $\mathbf{O}$, solid line, diabetic subjects (newly-diagnosed and known diabetic). See text for statistics

also present in the NDM and KDM groups. As assessed by logistic regression, the relative risk of having low SDNN, LF power, HF power, EI difference, BRS or RRmax was about 1.7 to 2.8 per 10 -year age difference in the non-diabetic group. The associations with sex were only present for SDNN [relative risk of 3.0 (1.0-8.6) for women] and RRmax [relative risk of 4.7 (1.6-14.0) for women] in the KDM group. Within the NGT group an association between $\mathrm{HbA}_{1 \mathrm{c}}$ and SDNN [relative risk of 2.1 (1.1-3.9) per $\% \mathrm{HbA}_{1 \mathrm{c}}$ ] and BRS [relative risk $2.2(1.1-4.1)$ per $\%$ $\mathrm{HbA}_{1 \mathrm{c}}$ ] was found. The associations of measures of cardiovascular autonomic function with cardiovascular disease were rather weak or absent in the separate glucose tolerance groups. The associations of EI difference and RRmax with coronary artery disease as observed in the total group were not statistically significant in the separate glucose tolerance groups. The observed relation of Mean NN with cerebrovascular disease was present only in the NGT group [relative risk of 3.7 (1.1-12.7)], whereas in the IGT, NDM and KDM groups the relative risks were 1.5, 1.1, and 2.2 , respectively. For the EI difference an association with cerebrovascular disease was also found in the NGT group, relative risk of 5.1 (1.4-18.7). When taking coronary artery, cerebrovascular and peripheral arterial disease together (any cardiovascular disease), no substantial relations were found in the separate glucose tolerance groups. The observed relation with the use of anti-hypertensive drugs was, for SDNN, [relative risk 3.1 (1.5-6.3) for users vs non-users] present in the IGT group, for LF power [relative risk of 4.3 (2.0-9.1)] also in the IGT group, for EI difference [relative risk of $2.1(1.1-4.4)]$ in the NGT group and for BRS [relative risk of 2.6 (1.3-5.4)] also in the NGT group. For RRmax/min, all glucose tolerance groups showed a relative risk of about 1.5 for users of anti-hypertensive drugs vs non-users, but this was not statistically significant for any of the glucose tolerance groups separately. The observed association of insulin with Mean NN [relative risk of 2.7 (1.4-5.0) per $50 \mathrm{pmol} / \mathrm{l}]$ was present in the NDM group and not present in any of the other groups (relative risk approximately 1); and for RRmax/min [relative risk of $1.5(0.97-2.3)$ per $50 \mathrm{pmol} / \mathrm{l}]$ the association was marginally statistically significant in the KDM group and again not in any of the other groups. The observed association in the complete group with physical activity could not be shown in any of the glucose tolerance groups separately.

The associations as observed for hypertension on the basis of blood pressure, BMI, and WHR by linear regression analysis in the separate glucose tolerance groups were not statistically significant in logistic regression (this was possibly due to the fact that logistic regression is less sensitive than linear regression when using continuous variates which show a clear linear association). Thus, only linear regression showed that hypertension on the basis of blood pressure was statistically significantly associated with Mean NN and HF power in the IGT group; BMI was statistically significantly associated with LF power, $\mathrm{LF} /(\mathrm{LF}+\mathrm{HF})$, EI difference in the NDM group, with RRmax/min in the IGT group, and with SBP difference in the IGT, NDM, and KDM group; WHR was statistically significantly associated with LF power and the $\mathrm{LF} /(\mathrm{LF}+\mathrm{HF})$ in the NGT group.

Full model (for each glucose tolerance group). Finally, all putative determinants were entered stepwise into one logistic regression model for each glucose tolerance group separately. Again, effect modification was confirmed as being statistically significant by adding the appropriate product terms in the regression models (for the complete group). In general, the results were very similar to the results as reported in the section above: (1) the same putative determinants were marked as statistically significant, and (2) also the size of the relative risks was about the same, indicating that no major confounding between cardiovascular, metabolic and life-style factors was present. Only in the NDM and KDM groups did life-style factors, notably smoking and consumption of alcoholic beverages, turn out to be statistically significant after adjustment for cardiovascular disease for some of the cardiovascular autonomic function measures. The estimated relative risks, however, were sometimes below one and had very wide confidence intervals. 


\section{Discussion}

In this study group of 50- to 75-year-old subjects, age, sex, glucose tolerance, waist-to-hip ratio, use of antihypertensive drugs and insulin were associated with cardiovascular autonomic function.

We showed that diabetes is the strongest determinant of autonomic function. All autonomic measures, except LF/(LF + HF), showed a consistent effect: increased risk for low autonomic function with decreasing glucose tolerance. These observations are in line with previous studies [1, 5, 10-12]. In addition, the association between autonomic function test measures and the glycaemic index $\mathrm{HbA}_{1 \mathrm{c}}$ in the NGT group has not been reported previously. A relation between the HF power, a marker of vagal function, and serum insulin and glucose has been reported [22,23]. We observed that in the NGT group the SDNN and BRS were statistically significantly associated with $\mathrm{HbA}_{1 \mathrm{c}}$.

Our study confirmed that age and sex are determinants of all autonomic function test measures [13-16]. This finding, however, is new for subjects aged 50 to 75 years. Furthermore, the age dependency was mainly present in the non-diabetic group. One explanation could have been the slightly younger age of these persons compared with the diabetic subjects, since the steepness of the relation between age and autonomic function decreases with age. The range of ages, however, was about the same for non-diabetic and diabetic subjects, indicating that it was truly an effect of diabetes (Fig.1). This effect modification was also statistically tested in a model including age, sex and diabetes, and the product term age $\mathrm{x}$ diabetes yielded a $p$-value of 0.065 .

Various cardiovascular diseases, including congestive heart failure, myocardial infarction, and hypertension have been reported to influence cardiovascular reflexes $[18,19]$. In this study, the available measure of cardiovascular disease was not a strong determinant among the general population. Only for coronary artery disease were some associations observed. It cannot be excluded, however, that more specific measurement of subclinical cardiovascular disease by means of, for example, cardiography, may be associated with low autonomic function. We did observe associations with blood pressure; however, after adjusting for cardiovascular disease and use of antihypertensive drugs, high blood pressure ( $\geq 160 / 95 \mathrm{mmHg}$ ) was not a major determinant. Currently hypertension is defined as a blood pressure over 140/90. At the time of the measurements (1989-1992) only blood-pressure values over 160/ $95 \mathrm{mmHg}$ were treated, and therefore the results of these cut-off points were presented. In addition, we examined whether changing the definition to the currently accepted values of 140/90 would affect our results but this did not affect the results (data not shown).
Also several drugs prescribed for cardiovascular disease, including beta-blocking agents, have been found to influence the autonomic nervous system [21]. In our study the strongest associations were observed for SDNN, LF power, LF/(LF + HF), EI difference, and RRmax/min. The latter is possibly explained by the fact that many anti-hypertensive drugs are peripheral sympathetic vasomotor blocking agents. These attenuate sympathetic vasomotor regulation, which is one of the strongest effector mechanisms of baroreflex blood pressure control. A widely accepted theory about the origin of low frequency (around $0.1 \mathrm{~Hz}$ ) oscillations in $\mathrm{BP}$ and HR is the theory of a resonating baroreflex closed control loop caused by certain delays in vasomotor control [36, 37]. According to this theory, spontaneous or respiration-related fluctuations in blood pressure and heart rate around this resonance frequency of $0.1 \mathrm{~Hz}$ will be amplified in normal conditions. In conditions of diminished vascular resistance control, however, e.g. due to sympathetic blocking agents, the closed loop resonance will disappear [36]. Therefore, autonomic function measures based on these $0.1 \mathrm{~Hz}$ variations, such as $\mathrm{LF}$ power, $\mathrm{LF} /(\mathrm{LF}+\mathrm{HF})$, EI difference, and SDNN, are expected to be associated with the use of, for instance, beta-blocking agents. On the other hand, the BRS parameter based on the ratio of HR to $B P$ variations will not be influenced by vasomotor active agents according to this resonance theory. This is supported by our findings. The association of cardiovascular autonomic function with the use of anti-hypertensive drugs was stronger in the non-diabetic group, possibly due to the fact that diabetic subjects already had low scores and the use of anti-hypertensive drugs had little additional impact.

Associations between indicators of obesity and cardiovascular autonomic dysfunction have been reported [17]. We also found an association between a high waist-to-hip ratio and low autonomic function in the complete group, and this relationship was also present in the glucose tolerance groups separately, but not consistently stronger in diabetic vs non-diabetic subjects. It has recently been reported that autonomic neuropathy (defined by a diminished E/I ratio) 5 years after the diagnosis of Type II diabetes was associated with an unfavourable metabolic risk profile that is part of the insulin resistance syndrome [23]. Although we did not observe an association of autonomic function measures with lipid concentrations, we did find a relation with fasting insulin, especially in diabetic subjects and both sympathetic and parasympathetic measures were affected, as has been reported before [38]. Since it is known that high concentrations of insulin may not be a marker of insulin resistance in diabetic subjects [39], a more likely explanation for our finding could be an effect of insulin on the activity of the sympathetic nerves: insulin infusions (euglycaemic clamp) have been 
shown to increase plasma norepinephrine concentrations (indicative of sympathetic functioning) in normal man [40]. Concomitantly with this sympathetic activation, the parasympathetic nervous function could be slowed down. Clearly, further studies are needed to enlighten the pathway through which insulin modulates autonomic functioning in diabetic patients.

Life-style factors have been reported to affect cardiovascular autonomic function [25, 26]. In our study only weak and less consistent associations were observed.

Limitations of the study. Our study, being an observational epidemiologic study possibly has potential biases. Matters of concern are the selection of the study group and possible misclassification.

Our study group consisted of an age-, sex- and glucose-tolerance-stratified group of people from an unselected general population. Thus a large contrast in glucose tolerance was obtained. Because no selection with regard to other putative determinants of cardiovascular autonomic function was made, the contrast in these other determinants was less optimal. This reduces the efficiency of the analyses. The moderate reproducibility of the test measures may lead to misclassification, thus reducing the power of the study. Test measures involving the measurement of SBP were least reproducible (data not shown). It is not likely, however, that the misclassification due to the lack of reproducibility is systematic, and therefore it will only affect the precision of the reported associations as assessed by linear regression and not the magnitude of the coefficient. For logistic regression the odds ratio will be underestimated due to eventual non-systematic misclassification.

The question remains whether the observed determinants are only correlates of cardiovascular autonomic function or whether they are associated with cardiovascular autonomic function in a causal way. This cannot be decided on the basis of these crosssectional results. From the literature it is known that lower values of cardiovascular autonomic function tests per se are an important finding, since they have prognostic value regarding cardiovascular death. This has been shown for healthy persons $[8,9]$, and for patients after a myocardial infarction [6]. Whether this relation between survival and autonomic function is dependent on glucose tolerance remains to be investigated.

In conclusion, in a general elderly population, measures of cardiovascular autonomic function are consistently associated with age, sex, glucose tolerance, waist-to-hip ratio, use of anti-hypertensive drugs and insulin, but less with cardiovascular disease and not with lipid profiles. Age and use of anti-hypertensive drugs were most strongly associated with cardiovascular autonomic function test measures in the non-diabetic population. For insulin the most pronounced association was observed in subjects with diabetes.

Acknowledgements. This study was supported by a grant from the Dutch Diabetes Research Foundation.

\section{References}

1. Ewing DJ, Martyn CN, Young RJ, Clarke BF (1985) The value of cardiovascular autonomic function tests: 10 years experience in diabetes. Diabetes Care 8: 491-498

2. Wieling W, van Brederode JF, de Rijk LG, Borst C, Dunning AJ (1982) Reflex control of heart rate in normal subjects in relation to age: a data base for cardiac vagal neuropathy. Diabetologia 22: 163-166

3. Mackay JD, Page MM, Cambridge J, Watkins PJ (1980) Diabetic autonomic neuropathy. The diagnostic value of heart rate monitoring. Diabetologia 18: 471-478

4. Bellavere F, Balzani I, De Masi G et al. (1992) Power spectral analysis of heart-rate variations improves assessment of diabetic cardiac autonomic neuropathy. Diabetes 41: 633-640

5. Weston PJ, James MA, Panerai R et al. (1996) Abnormal baroreceptor-cardiac reflex sensitivity is not detected by conventional tests of autonomic function in patients with insulin-dependent diabetes mellitus. Clinical Science 91: 59-64

6. La Rovere MT, Bigger JT Jr, Marcus FI, Mortara A, Schwartz PJ (1998) Baroreflex sensitivity and heart-rate variability in prediction of total cardiac mortality after myocardial infarction. Lancet 351: 478-484

7. Bigger JT, Fleiss JL, Rolnitzky LM, Steinman RC (1993) The ability of several short-term measures of RR variability to predict mortality after myocardial infarction. Circulation 88: 927-934

8. Dekker JM, Schouten EG, Klootwijk P, Pool J, Swenne CA, Kromhout D (1997) Heart rate variability from short electrocardiographic recordings predicts mortality from all causes in middle-aged and elderly men -the Zutphen Study. Am J Epidemiol 145: 899-908

9. Tsuji H, Venditti FJ, Jr., Manders ES et al. (1994) Reduced heart rate variability and mortality risk in an elderly cohort. The Framingham Heart Study. Circulation 90: 878-883

10. Freeman R, Saul JP, Roberts MS, Berger RD, Broadbridge C, Cohen RJ (1991) Spectral analysis of heart rate in diabetic autonomic neuropathy. A comparison with standard tests of autonomic function. Archives of Neurology 48: 185-190

11. Ziegler D, Dannehl K, Volksw D, Muhlen H, Spuler M, Gries FA (1992) Prevalence of cardiovascular autonomic dysfunction assessed by spectral analysis and standard tests of heart-rate variation in newly diagnosed IDDM patients. Diabetes Care 15: 908-911

12. Frattola A, Parati G, Gamba P et al. (1997) Time and frequency domain estimates of spontaneous baroreflex sensitivity provide early detection of autonomic dysfunction in diabetes mellitus. Diabetologia 40: 1470-1475

13. O'Brien IA, O'Hare P, Corrall RJ (1986) Heart rate variability in healthy subjects: effect of age and the derivation of normal ranges for tests of autonomic function. Br Heart J 55: 348-354

14. Ziegler D, Laux G, Dannehl K et al. (1992) Assessment of cardiovascular autonomic function: age-related normal ranges and reproducibility of spectral analysis, vector anal- 
ysis, and standard tests of heart rate variation and blood pressure responses. Diabet Med 9: 166-175

15. James MA, Robinson TG, Panerai RB, Potter JF (1996) Arterial baroreceptor-cardiac reflex sensitivity in the elderly. Hypertension 28: 953-960

16. Huikuri HV, Pikkujamsa SM, Airaksinen KE et al. (1996) Sex-related differences in autonomic modulation of heart rate in middle-aged subjects. Circulation 94: 122-125

17. Gelber DA, Pfeifer M, Dawson B, Schumer M (1997) Cardiovascular autonomic nervous system tests: determination of normative values and effect of confounding variables. J Auton Nerv Syst 62: 40-44

18. Osterziel KJ, Hanlein D, Willenbrock R, Eichhorn C, Luft F, Dietz R (1995) Baroreflex sensitivity and cardiovascular mortality in patients with mild to moderate heart failure. Br Heart J 73: 517-522

19. Lucini D, Pagani M, Mela GS, Malliani A (1994) Sympathetic restraint of baroreflex control of heart period in normotensive and hypertensive subjects. Clinical Science 86: 547-556

20. Salo TM, Viikari JS, Antila KJ, Voipio-Pulkki LM, Jalonen JO, Valimaki, IA. (1996) Antihypertensive treatment and heart rate variability in diabetic patients: role of cardiac autonomic neuropathy. J Auton Nerv Syst 60: 61-70

21. Aronson D (1997) Pharmacologic modulation of autonomic tone - implications for the diabetic patient. Diabetologia 40: 476-481

22. Liao D, Cai J, Brancati FL et al. (1995) Association of vagal tone with serum insulin, glucose, and diabetes mellitus - The ARIC Study. Diabetes Res Clin Pract 30: 211-221

23. Gottsater A, Ahmed M, Fernlund P, Sundkvist G (1999) Autonomic neuropathy in Type 2 diabetic patients is associated with hyperinsulinaemia and hypertriglyceridaemia. Diabet Med 16: 49-54

24. Bonaa KH, Arnesen E (1992) Association between heart rate and atherogenic blood lipid fractions in a population. The Tromso Study. Circulation 86: 394-405

25. Niedermaier ON, Smith ML, Beightol LA, ZukowskaGrojec Z, Goldstein, DS, Eckberg DL (1993) Influence of cigarette smoking on human autonomic function. Circulation 88: 562-571

26. Seals DR, Chase PB (1989) Influence of physical training on heart rate variability and baroreflex circulatory control. J Appl Physiol 66: 1886-1895

27. Stamler J, Vaccaro O, Neaton JD, Wentworth D (1993) Diabetes, other risk factors, and 12-yr cardiovascular mortality for men screened in the Multiple Risk Factor Intervention Trial. Diabetes Care 16: 434-444

28. DeVegt E, Dekker JM, Ruhé HG et al. (1999) Hyperglycaemia is associated with all-cause and cardiovascular mor- tality in the Hoorn population: the Hoorn Study. Diabetologia 42: 926-931

29. DeNeeling JND, Beks PJ, Bertelsmann FW, Heine RJ, Bouter LM (1996) Peripheral somatic nerve function in relation to glucose tolerance in an elderly Caucasian population - the Hoorn Study. Diabet Med 13: 960-966

30. World Health Organization Study Group on Diabetes Mellitus (1985) Technical Report Series No 727, WHO, Geneva

31. Rose GA, Blackburn H, Gillum RF, Prineas RJ (1982) Cardiovascular Survey Methods, 2nd edn. Monograph Series No 56, WHO, Geneva

32. Beks PJ, Mackaay AJ, DeNeeling JN, de Vries H, Bouter, LM, Heine RJ (1995) Peripheral arterial disease in relation to glycaemic level in an elderly Caucasian population: the Hoorn study. Diabetologia 38: 86-96

33. Task Force of the European Society of Cardiology and the North American Society of Pacing and Electrophysiology (1996) Heart rate variability: standards of measurement, physiological interpretation and clinical use. Circulation 93: 1043-1065

34. Faes TJ, DeNeeling NN, Kingma R, TenVoorde BJ, Karemaker JM (1995) On the quantification of heart rate changes in autonomic function tests: relations between measures in beats per minute, seconds and dimensionless ratios. Clinical Science 89: 557-564

35. Bendat JS, Piersol AG (1986) Random data analysis and measurement procedures, 1st edn. John Wiley and Sons, New York, Chichester, Brisbane, Toronto, Singapore

36. TenVoorde BJ, Faes TJC, Janssen TWJ, Scheffer GJ, Rompelman O (1995) Respiratory modulation of blood pressure and heart rate studied with a computer model of baroreflex control. In: Di Rienzo M, Mancia G, Parati G, Pedotti A, Zanchetti A (eds) Computer analysis of cardiovascular signals. IOS Press, Amsterdam, Oxford, Tokyo, Washington, pp 119-134

37. Baselli G, Cerutti S, Civardi S, Malliani A, Pagani M (1988) Cardiovascular variability signals: towards the identification of a closed-loop model of the neural control mechanisms. IEEE Trans Biomed Eng 35: 1033-1046

38. Toyry JP, Niskanen LK, Mantysaari MJ, Lansimies EA, Uusitupa MI (1996) Occurrence, predictors, and clinical significance of autonomic neuropathy in NIDDM. Tenyear follow-up from the diagnosis. Diabetes 45: 308-315

39. Laakso M (1993) How good a marker is insulin level for insulin resistance? Am J Epidemiol 137: 959-965

40. Rowe JW, Young JB, Minaker KL, Stevens AL, Pallotta J, Landsberg L (1981) Effect of insulin and glucose infusions on sympathetic nervous system activity in normal man. Diabetes 30: 219-225 